Genome Insight

Genetics of Microorganisms

\title{
Genomic sequencing of two isolates of Ralstonia solanacearum causing Sergipe facies and comparative analysis with Bugtok disease isolates
}

\author{
Jéssica Rodrigues da Silva ${ }^{1}$, Ana Karolina Leite Pais ${ }^{2}$, Greecy Mirian Rodrigues Albuquerque ${ }^{2}$, \\ Adriano Márcio Freire Silva ${ }^{3}$, Wilson José Silva Junior ${ }^{4}$, Valdir de Queiroz Balbino, \\ Maria Esther Noronha Fonseca ${ }^{5}$, Marco Aurélio Siqueira da Gama ${ }^{2}$, Elineide Barbosa de Souza ${ }^{6}$ \\ and Rosa de Lima Ramos Mariano ${ }^{2}$ \\ ${ }^{I}$ Instituto Federal de Educação, Ciência e Tecnologia do Piauí-IFPI, Teresina, PI, Brazil. \\ ${ }^{2}$ Universidade Federal Rural de Pernambuco - UFRPE, Departamento de Agronomia, Recife, PE, Brazil. \\ ${ }^{3}$ Universidade Federal de Alagoas - UFAL, Departamento de Agronomia, Maceió, AL, Brazil. \\ ${ }^{4}$ Universidade Federal de Pernambuco - UFPE, Departamento de Genética, Recife, PE, Brazil. \\ ${ }^{5}$ Embrapa Hortaliças, Brasília, DF, Brazil. \\ ${ }^{6}$ Universidade Federal Rural de Pernambuco - UFRPE, Departamento de Biologia, Recife, PE, Brazil.
}

\begin{abstract}
Ralstonia solanacearum is the causal agent of Moko disease in bananas, which in the state of Sergipe in northeastern Brazil causes "Sergipe facies". This disease induces atypical symptoms similar to those of Bugtok disease in the Philippines. This study was conducted to sequence, assemble, and annotate the genomes of the Sergipe faciescausing isolates SFC and IBSBF2570 (sequevar IIA-53) and compare their genomes with two representative isolates causing Bugtok disease. The genomes were sequenced and assembled, resulting in lengths of $5.58 \mathrm{Mb}$ (SFC) and $5.46 \mathrm{Mb}$ (IBSBF2570) in 185 and 174 contigs, respectively. The isolates of Sergipe facies and Bugtok disease showed similarities in their gene contents. We identified 5,668 information clusters, 3,752 of which were shared by all genomes (core genes). Moreover, 3,585 single-copy genes were identified. Isolates causing Bugtok disease exclusively shared 266 more information clusters than the isolates causing Sergipe facies. These results suggest that Sergipe facies and Bugtok disease isolates show high genomic similarity. However, the similarity is even greater between the Bugtok disease isolates. This may be because of their longer period of interaction compared to Sergipe facies isolates.
\end{abstract}

Keywords: Banana tree, inflorescence infection, phylotype.

Received: May 19, 2020; Accepted: September 21, 2020.

Ralstonia solanacearum is the causal agent of Moko disease in triploid bananas and Heliconia species (Hayward, 1994). Because of its heterogenicity, it is considered a species complex (Fegan and Prior, 2005) and has been classified into four phylotypes based on the geographical origins of the isolates and several sequevars, which are groups of strains with highly conserved regions in the endoglucanase gene (Fegan and Prior, 2006). Recently, three species were proposed in this species complex: $R$. solanacearum, $R$. pseudosolanacearum, and $R$. syzygii (Safni et al., 2014). The isolates causing Moko in bananas were classified as $R$. solanacearum belonging to phylotype II and subdivided into IIA and IIB (Fegan and Prior, 2006), originating in the Americas (Prior and Fegan, 2005), and sequevars IIA-6, IIA-24, IIA-41, IIA-53, IIB-3, IIB-4, and IIB-25 (Fegan and Prior, 2005; 2006; Albuquerque et al., 2014).

Typical symptoms of Moko include yellowing and withering of the leaves, caused by an infection that begins in the rhizomes and moves towards the pseudostem. The fruits become deformed, black, and stunted. Banana plants close to maturity may not display any obvious symptoms but the internal fruit pulp can still show dry rot and the plants may

Send correspondence to Elineide Barbosa de Souza. Universidade Federal Rural de Pernambuco - UFRPE, Departamento de Biologia, AV. D. Manoel de Medeiros, s/n Dois Irmãos, 52171-900, Recife - PE, Brazil. E-mail: elineidebs@yahoo.com.br. die. However, in the state of Sergipe in northeastern Brazil, there is a variant of Moko disease known as "Sergipe facies". This disease is caused by endemic isolates of $R$. solanacearum within sequevar IIA-53, which initiate symptoms in the inflorescences and progress to dry rot and fruit deformation without wilting. These symptoms are similar to those caused by Bugtok disease, which occurs in the Philippines (Albuquerque et al., 2014). The latter mainly attacks the banana cultivars 'Saba' and 'Cardaba' and is associated with isolates from sequevar IIB-3 (Thwaites et al., 2000).

The objective of this study was to sequence, assemble, and annotate the genomes of two isolates of sequevar IIA53 of $R$. solanacearum, the causal agent of Sergipe facies (SFC and IBSBF2570), compare them with the genomes of isolates of sequevar IIB-3, the causal agent of Bugtok disease (CIP417 and Molk2), and deposit these sequences into the United States National Center for Biotechnology Information (NCBI) database.

The SFC and IBSBF2570 isolates were grown in 2,3,5-triphenyl tetrazolium chloride medium (Kelman, 1953) at $28^{\circ} \mathrm{C}$ for $48 \mathrm{~h}$, and then DNA was extracted using the PureLink ${ }^{\circledR}$ Genomic DNA kit (Thermo Fisher Scientific, Waltham, MA, USA) following the manufacturer's instructions. The DNA was quantified using a spectrophotometer (BioDrop, Thermo Fisher Scientific) and subjected to electrophoresis on $0.8 \%$ agarose gels to assess its integrity. 
Sequencing libraries were prepared using the Illumina Nextera DNA Flex Prep Kit (Illumina, San Diego, CA, USA). Sequencing was performed on a MiSeq-2500 Platform (Illumina) to generate pair-ended reads at the ESALQ-USP Functional Genomics Center. The quality of the reads was verified using FastQC (Andrews, 2010), sequence filtering was performed using FASTX-Clipper v. 0.0.13, and sequence trimming was performed using Sickle v. 1.33 (Joshi and Fass, 2011).

De novo assembly was performed using the Unicycler pipeline (Wick et al., 2017) and ABACAS software v.1.3.1 (Assefa et al., 2009) for alignment with the PROmer and NUCmer algorithms. The complete genomes of the UW163 (GCF_001587135.1) and Po82 (GCF_000215325.1) isolates (both sequevar IIB-4) of $R$. solanacearum were used as alignment references. Contig evaluation and selection of the scaffolds formed from alignments showing the lowest number of Ns and the largest number of predicted genes were performed using QUAST software v.5.0.2 (Gurevich et al., 2013). BUSCO software (Seppey et al., 2019; implemented in QUAST) was used to identify single-copy orthologs and analyze the conservation of the gene contents. The alignment between the Sergipe facies (SFC and IBSBF2570) and Bugtok (CIP417 and Molk2) causing isolates genomes was visualized using the Circos package (Krzywinski et al., 2009 ) in QUAST. Gene predictions and automatic annotation of proteins present in the assembled scaffolds were performed using the RAST platform (Aziz et al., 2008), followed by analysis of orthologous gene clusters using OrthoVenn (Xu et al., 2019).
After treatment of the raw reads, the genomes were assembled at the contig level to generate sequences of 5.5 $\mathrm{Mb}$ (SFC) and 5.4 Mb (IBSBF2570) in 185 and 174 contigs, respectively (Table 1). The assembly showed little variation in N50 (i.e., the shortest contig length required for 50\% genome coverage) between the genomes and a high rate of conservation in the gene content (98\%), indicating a reliable assembly. The lengths of the sequences and their GC content (66\%) were consistent with the genome sequences of the Mokocausing isolates deposited in the NCBI database. Scaffold construction was based on the PROmer algorithm using the $R$. solanacearum isolates Po82 and UW163 as references for the chromosome and megaplasmid, respectively.

Based on the annotation of the isolates causing Sergipe facies, proteins were distributed into functional groups, and approximately $65 \%$ of the encoded proteins concentrated into seven subsystems: I- amino acids and derivatives (17.8\%); II- carbohydrates (10.1\%); III- cofactors, vitamins, protein groups, and pigments (10.0\%); IV- protein metabolism $(8.4 \%)$; V- membrane transport (8.3\%); VI- fatty acids, lipids, and isopropenoids (5.5\%); and VII- breathing (5.2\%). Isolates causing Bugtok disease showed similar results, with a slightly different order of subsystem representativeness: I- amino acids and derivatives (16.7\%); II- carbohydrates (10.3\%); III- membrane transport $(9.6 \%)$; IV- cofactors, vitamins, protein groups, and pigments $(9.6 \%)$; V- protein metabolism (9.5\%); VI- breathing (5.0\%); and VII- fatty acids, lipids, and isopropenoids (4.2\%; Figure 1). Because of the similarities between the atypical symptoms of the Sergipe facies and

Table 1 - Genome characteristics of isolates SFC and IBSBF2570 of Ralstonia solanacearum causing Sergipe facies disease.

\begin{tabular}{|c|c|c|c|c|}
\hline Features & \multicolumn{2}{|c|}{$\mathrm{SFC}$} & \multicolumn{2}{|c|}{ IBSBF 2570} \\
\hline Coverage & \multicolumn{2}{|c|}{151.35} & \multicolumn{2}{|c|}{156.75} \\
\hline Genomes size $(b p)^{\mathrm{a}}$ & \multicolumn{2}{|c|}{5.578 .372} & \multicolumn{2}{|c|}{5.455 .860} \\
\hline $\mathrm{G}+\mathrm{C}$ content $(\%)$ & \multicolumn{2}{|c|}{66.61} & \multicolumn{2}{|c|}{66.59} \\
\hline Total contig number & \multicolumn{2}{|c|}{185} & \multicolumn{2}{|c|}{174} \\
\hline N50 & \multicolumn{2}{|c|}{94.771} & \multicolumn{2}{|c|}{94.853} \\
\hline BUSCO (\%) & \multicolumn{2}{|c|}{98.65} & \multicolumn{2}{|c|}{98.65} \\
\hline Coding sequence & \multicolumn{2}{|c|}{4,937} & \multicolumn{2}{|c|}{4,862} \\
\hline Subsytems number & \multicolumn{2}{|c|}{351} & \multicolumn{2}{|c|}{345} \\
\hline RNAs & \multicolumn{2}{|c|}{52} & \multicolumn{2}{|c|}{51} \\
\hline \multirow[t]{2}{*}{ Genome size $(b p)^{b}$} & \multicolumn{2}{|c|}{5.722 .671} & \multicolumn{2}{|c|}{5.713 .471} \\
\hline & Chrom $^{\mathrm{c}}$ & Plasmid $^{\mathrm{d}}$ & Chrom & Plasmid \\
\hline Reference isolates & ${ }^{\mathrm{e}} \mathrm{Po} 82$ & eUW163 & Po82 & UW163 \\
\hline Genome size $(b p)^{b}$ & 3.630 .670 & 2.092 .001 & 3.656 .700 & 2.056 .771 \\
\hline
\end{tabular}

${ }^{\text {a }}$ Genome size before alignment;

${ }^{\mathrm{b}}$ Genome size after alignment;

${ }^{c}$ Chromosome;

${ }^{\mathrm{d}}$ Megaplasmid;

${ }^{\text {e }}$ Po82 e UW163 (sequevar IIB-4) - Isolates of $R$. solanacearum used as a reference in the alignment of genomic sequences. 

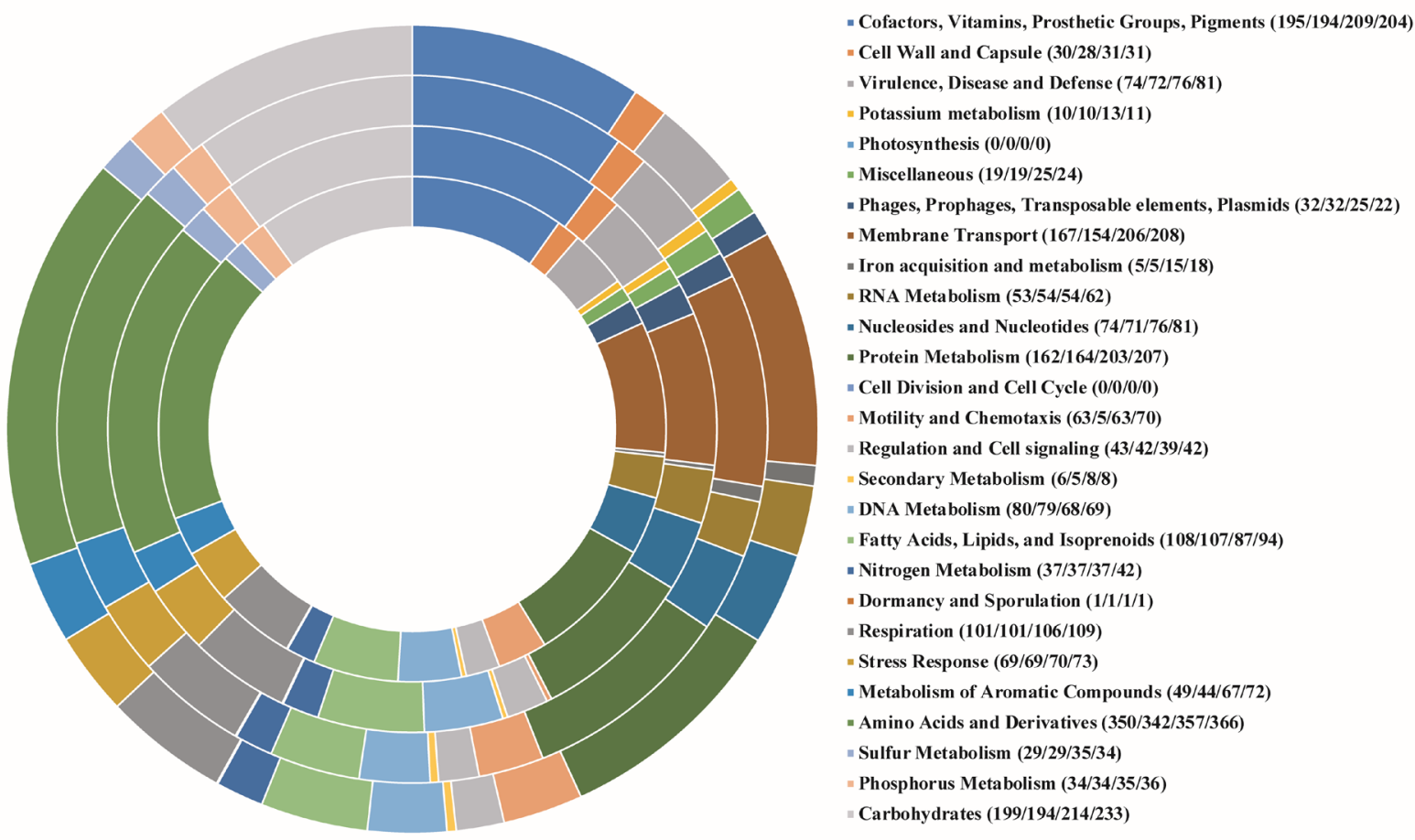

Figure 1 - Distribution of the subsystem category and gene coverage of isolates of Ralstonia solanacearum causing Sergipe facies (SFC and IBSBF2570) and Bugtok disease (CIP417 and Molk2). The graphic representation shows the grouping of genes from the SFC, IBSBF2570, CIP417, and Molk2 genomes from the inside to out. The numbers between parentheses in the legend represent the number of genes in each subsystem in the same order.

Bugtok diseases, which initiate from inflorescences in both cases, a high degree of genome similarity was expected.

Visualization of the alignment built based on the genomic sequences of the two reference isolates (Po82 and UW163) revealed the absence of GC content in two regions of Po82 (Figure 2A, outermost circle) and four regions of UW163 (Figure 2B, outermost circle) in all isolates. The assembly strip individually highlighted the incompatibility (taller columns $=$ more incompatibility) and gene density (higher green or red intensity $=$ higher gene density) of all isolates, enabling identification of low-density regions in the isolates causing Sergipe facies (SFC and IBSBF2570). This was not observed in isolates causing Bugtok disease, nor was it correlated with gene density (Figure 2).

In a Venn diagram of the isolates causing Sergipe facies and Bugtok disease, 5,668 information clusters were identified, of which 3,752 were shared between all genomes (core genes), and 3,585 were single copy genes (Figure 3; Table S1). The isolates that cause Bugtok disease symptoms exclusively shared $970(17.1 \%)$ clusters involved in 76 biological processes and 17 molecular functions and were associated with seven cellular components. The isolates that cause Sergipe facies shared 704 (12.4\%) clusters involved in 65 biological processes and 11 molecular functions and were associated with five cellular components (Table S1). The results indicate that the isolates causing Bugtok disease showed greater genetic similarity, as they exclusively shared 266 more information clusters than those causing Sergipe facies. The greater genetic similarity between Bugtok isolates may be related to the increased interaction time between the isolates, considering that Bugtok disease was first reported in 1965 (Soguilon et al., 1995, Thwaites et al., 2000), whereas Sergipe facies was first reported in 2014 (Albuquerque et al., 2014). However, other factors cannot be ruled out, particularly with regard to living organisms interacting freely in nature.

The sequencing, assembly, and annotation of the genomes of isolates of $R$. solanacearum causing Sergipe facies (sequevar IIA-53) provide a foundation for further research aiming to understand the interactions between $R$. solanacearum isolates and banana. SFC and IBSBF2570 are the only northeast Brazilian isolates and first isolates causing Sergipe facies whose genomes have been sequenced.

The genomes have been deposited in DDBJ/EMBL/ GenBank under accession numbers CP026090 and CP026091 (for the chromosome and megaplasmid of isolate IBSBF2570, respectively) and CP026092 and CP026093 (for the chromosome and megaplasmid of isolate SFC, respectively). 

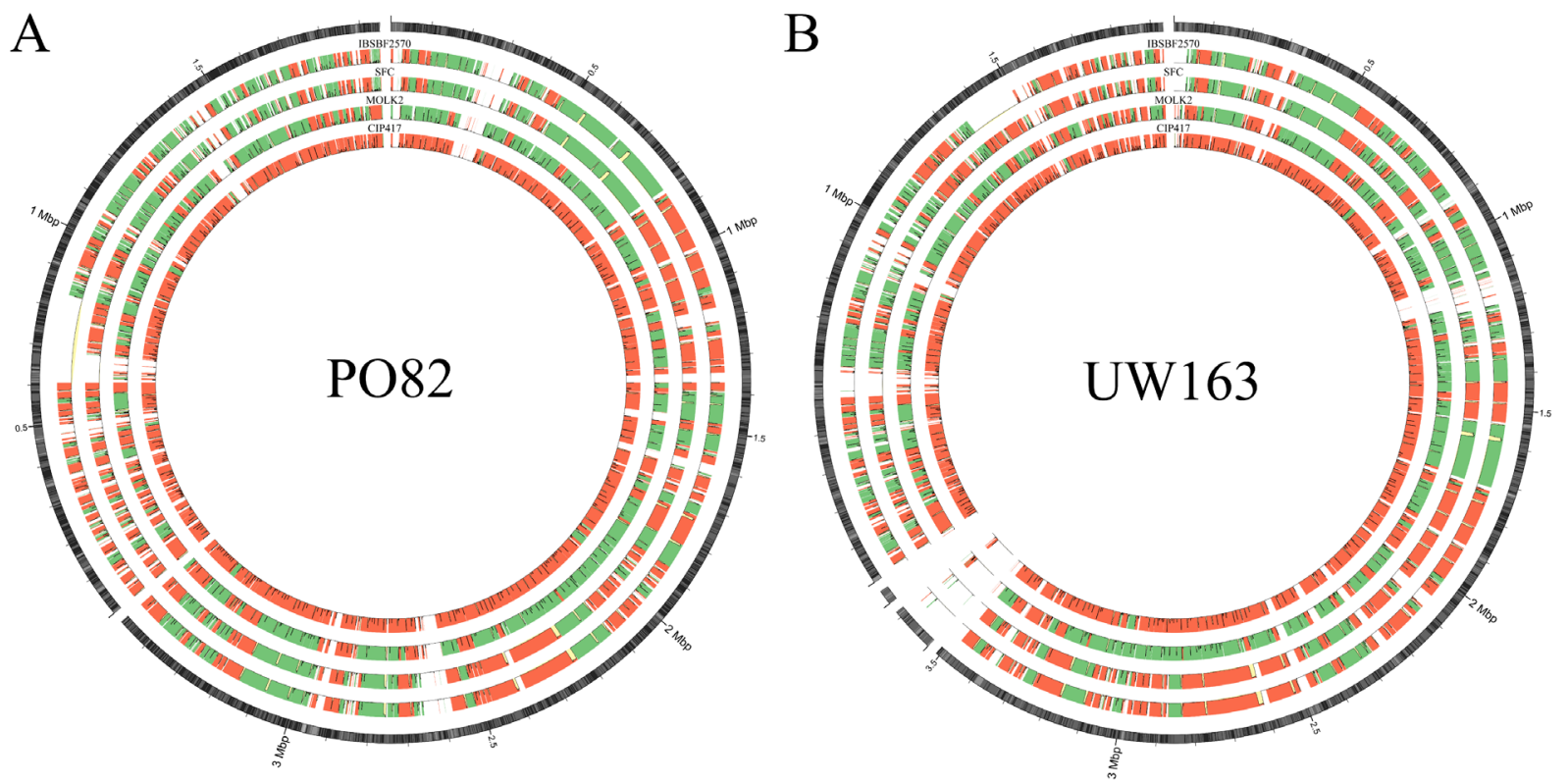

Figure 2 - Visualization of the alignment of Ralstonia solanacearum isolates causing Sergipe facies (SFC and IBSBF2570) and Bugtok disease (CIP417 and Molk2) with the reference isolates Po82 (A) and UW163 (B) of R. solanacearum. The outer circle represents the reference sequence with GC (\%) heatmap [from $0 \%$ (white) to $77 \%$ (black)]. Assembly tracks are combined with the display of incompatibilities: taller columns indicate a higher rate of incompatibility. Darker colors indicate higher gene density. The green color represents similar contigs to the reference and red color indicates misassembled contigs.
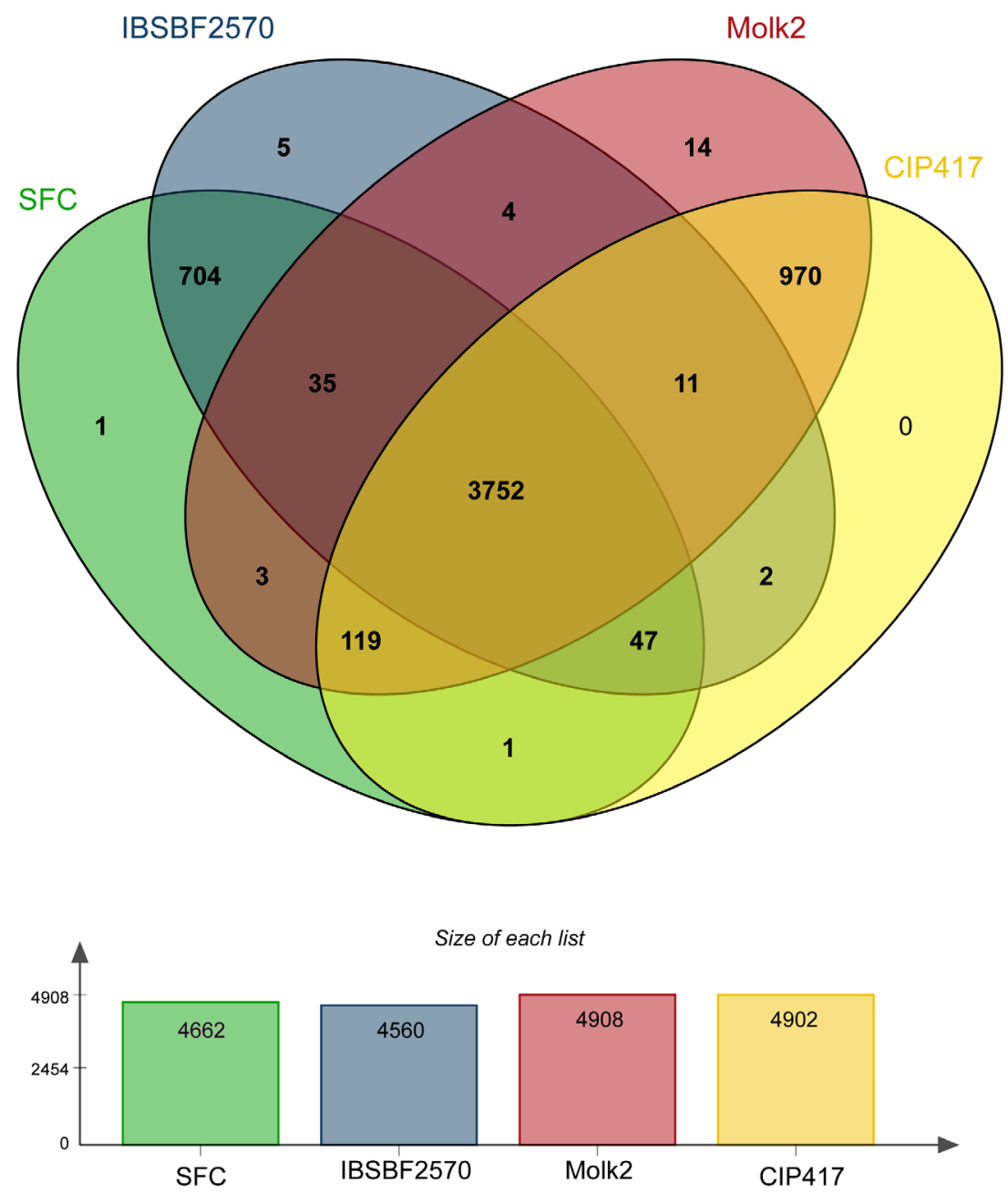

Figure 3 - Venn diagram showing clusters present in the genome of isolates of Ralstonia solanacearum causing Sergipe facies (SFC and IBSBF2570) and Bugtok disease (CIP417 and Molk2). 


\section{Acknowledgements}

We thank the Conselho Nacional de Ciência e Tecnologia, Brazil and Fundação de Amparo a Ciência e Tecnologia do Estado de Pernambuco, Brazil. We also thank the Laboratory of Bioinformatics and Evolutionary Biology of Universidade Federal de Pernambuco for making their servers available for bioinformatics analysis.

\section{Conflict of Interest}

The authors declare that there is no conflict of interest that could be perceived as prejudicial to the impartiality of the reported research.

\section{Authors Contributions}

RLRM, GMRA, AMFS and EBS conceived and designed the study, JRS, AKLP and WJSJ conducted the in silico analysis, JRS, AKLP, WJSJ, VQB, MENF and MASG analyzed the data, JRS, AKLP and GMRA wrote the manuscript with contributions from AMFS, WJSJ, MASG and EBS, all authors read and approved the final manuscript version.

\section{References}

Albuquerque GMR, Santos LA, Felix KCS, Rollemberg CL, Silva AMF, Souza EB, Cellier G, Prior P and Mariano RLR (2014) Moko disease-causing strains of Ralstonia solanacearum from Brazil extend known diversity in paraphyletic phylotype II. Phytopathology 104:1175-1182.

Andrews S (2010) FastQC: A quality control tool for high throughput sequence data. Babraham Bioinforma. DOI: citeulike-articleid:11583827.

Assefa S, Keane TM, Otto TD, Newbold C and Berriman M (2009) ABACAS: Algorithm-based automatic contiguation of assembled sequences. Bioinformatics 25:1968-9.

Aziz RK, Bartels D, Best AA, DeJongh M, Disz T, Edwards RA, Formsma K, Gerdes S, Glass EM, Kubal M et al (2008) The RAST Server: Rapid annotations using subsystems technology. BMC Genomics 9:e75.

Fegan M and Prior P (2005) How complex is the "Ralstonia solanacearum species complex". In: Allen C, Prior P and Hayward AC (eds) Bacterial wilt disease and the Ralstonia solanacearum Species Complex. MN: APS Press, St Paul, pp 449-61.

Fegan M and Prior P (2006) Diverse members of the Ralstonia solanacearum species complex cause bacterial wilts of banana. Australas Plant Pathol 35:93-101.

Gurevich A, Saveliev V, Vyahhi N and Tesler G (2013) QUAST: Quality assessment tool for genome assemblies. Bioinformatics 29:1072-1075.

Hayward AC (1994) The hosts of Pseudomonas solanacearum. In: Hayward AC and Hartman GL (Eds.). Bacterial Wilt the Disease and its Causative Agent, Pseudomonas solanacearum. CAB International, Wallingford, pp 9-24.

Joshi NA and Fass JN (2011) Sickle: A sliding-window, adaptive, quality-based trimming tool for FastQ files. (Version 1.33).
Kelman A (1953) The bacterial wilt caused by Pseudomonas solanacearum: a literature review and bibliography. Agricultural Experiment Technical Bulletin, North Carolina, $194 \mathrm{pp}$.

Krzywinski MI, Schein JE, Birol I, Connors J, Gascoyne R, Horsman D, Jones SJ and Marra MA (2009) Circos: An information aesthetic for comparative genomics. Genome Res 19:16391645.

Prior P and Fegan M (2005) Recent developments in the phylogeny and classification of Ralstonia solanacearum. Acta Hortic 695:127-136.

Safni I, Cleenwerck I, Vos PDe, Fegan M, Sly L and Kappler U (2014) Polyphasic taxonomic revision of the Ralstonia solanacearum species complex: proposal to emend the descriptions of Ralstonia solanacearum and Ralstonia syzygii and reclassify current $R$. syzygii strains as Ralstonia syzygii subsp. syzygi subsp. nov., $R$. solanacearum phylotype IV strains as Ralstonia syzygii subsp. indonesiensis subsp. nov., banana blood disease bacterium strains as Ralstonia syzygii subsp. celebesensis subsp. nov. and $R$. solanacearum phylotype I and III strains as Ralstonia pseudosolanacearum sp. nov. Int J Syst Evol Microbiol 64:3087-3103.

Seppey M, Manni M and Zdobnov EM (2019) BUSCO: Assessing genome assembly and annotation completeness. Methods $\mathrm{Mol}$ Biol 1962:227-245.

Soguilon CE, Magnaye LV and Natural MP (1995) Bugtok disease of banana. Inibap 33:4.

Thwaites R, Eden-Green SJ and Black R (2000). Diseases caused by bacteria. In: Jones DR (ed) Diseases of Banana, Abacá and Enset. CAB International, Wallingford, pp 213-239.

Wick RR, Judd LM, Gorrie CL and Holt KE (2017) Unicycler: Resolving bacterial genome assemblies from short and long sequencing reads. PLoS Comput Biol 13:1-22.

Xu L, Dong Z, Fang L, Luo Y, Wei Z, Guo H, Zhang G, Gu YQ, Coleman-Derr D, Xia Q et al (2019) OrthoVenn2: A web server for whole-genome comparison and annotation of orthologous clusters across multiple species. Nucleic Acids Res 47: W52-W58.

\section{Internet Resources}

On-line OrthoVenn, http://www.bioinfogenome.net/OrthoVenn (November 10, 2019).

On-line RAST, http://rast.theseed.org/FIG/rast.cgi (November 10, 2019).

\section{Supplementary material}

The following online material is available for this article: Table S1 - Annotation of orthologous genes present in the genomes of Ralstonia solanacearum isolates causing Sergipe facies (SFC and IBSBF2570) and Bugtok disease (CIP417 and Molk2).

Associate Editor: Ana Tereza R. Vasconcelos

License information: This is an open-access article distributed under the terms of the Creative Commons Attribution License (type CC-BY), which permits unrestricted use distribution and reproduction in any medium, provided the original article is properly cited. 